\title{
Once-daily aminoglycoside dosing: A new look at an old drug
}

A MINOGLYCOSIDES CONSTITUTE ONE OF THE OLDEST AND most versatile classes of antimicrobial agents in clinical use. Despite the addition of many new antimicrobials to our therapeutic armamentarium they remain a cornerstone of therapy in the treatment of serious bacterial infections $(1,2)$. Their major use has been for the treatment of suspected or proven Gramnegative bacillary infections and as empirical therapy of the febrile neutropenic patient. Juxtaposed with the acknowledged therapeutic efficacy of aminoglycosides has been the concern for aminoglycoside-induced renal and ototoxicity. These latter adverse effects have lead to dosing strategies that have sought to maintain serum aminoglycoside concentrations within a limited range of values. Over the years a great deal of investigative effort has been expended to facilitate our understanding of the pharmacokinetics and pharmacodynamics of aminoglycosides in order to ensure the achievement of therapeutic concentrations and to optimize their potent antibacterial activity while simultaneously lessening their toxicity (3-5). When selecting an aminoglycoside dosing regimen, attention must be paid to pharmacokinetic properties of the agent, microbiological function of the organism or likely organism(s) causing infection, site of infections, ease of administration, toxicity, clinical efficacy and cost containment.

Perusal of the literature on aminoglycoside dosing regimens accumulated over the past two decades reveals that the once-daily dosing of aminoglycosides may be associated with less toxicity while maintaining excellent therapeutic efficacy (6-9). This dosing approach is neither novel nor unique, but recent advances in pharmacokinetics and microbiology coupled with recent clinical trials on this issue and the necessity for improving quality care while reducing costs has created the opportunity for a paradigm shift in the manner in which aminoglycosides are dosed.

Once-daily dosing of aminoglycosides meets or exceeds all of the desirable attributes mentioned above with respect to the selection of a dosing regimen. With regard to the pharmacokinetic aspects of aminoglycosides, a narrow therapeutic index has been accepted as a hallmark for many years. Dosing strategies have sought to maintain serum aminoglycoside concentrations within a narrowly defined range of values in an attempt to optimize efficacy by exceeding the minimum inhibitory concentration (MIC) for the offending pathogen and to minimize toxicity by avoiding excessive concentrations. Guidelines for dosing adjustments with compromised renal function and the concept of closely monitoring serum aminoglycoside concentrations have become an accepted and routine practice in many hospital settings. The need to maintain aminoglycoside serum concentrations within such strict limits to maintain therapeutic efficacy and to reduce toxicity may be challenged on several fronts.

The aminoglycosides demonstrate a property known as concentration-dependent killing $(10,11)$. Clinical studies have demonstrated that achievement of high peak serum concentrations of the aminoglycoside relative to the MIC of the microorganism being treated may be a major determinant of the clinical response to the aminoglycosides. This optimization of the peak:MIC ratio can best be obtained by the once-daily administration of aminoglycosides that results in high peak concentrations of the drug (peak target is about 20 $\mathrm{mg} / \mathrm{L}$ ). In addition to concentration-dependent killing, aminoglycosides also demonstrate a property known as the postantibiotic effect (12), which may be defined as a period of time after complete removal of the antibiotic during which there is no growth of the target organism. Although once-daily dosing of aminoglycosides may result in a period of up to $12 \mathrm{~h}$ during which there are no detectable serum concentrations of the drug, the postantibiotic effect of the aminoglycosides allows for oncedaily dosing without compromising therapeutic efficacy.

\section{DRUG TOXICITY}

The major determinant of aminoglycoside-induced renal and ototoxicity is the accumulation of these agents within the renal cortex and the perilymph of the inner ear, respectively $(3,13)$. Uptake and accumulation of aminoglycosides into renal cortical tissue demonstrate saturable kinetics. The saturable feature of these kinetics make peak aminoglycoside concentrations irrelevant when considering tissue accumulation of the drug. Less frequent dosing of aminoglycosides allows for serum concentrations of the drug to fall well below the threshold for binding to tissue receptors, also allowing for the back-diffusion of the aminoglycosides from 
the renal cortex and inner ear, which may theoretically limit drug toxicity. In animal models, rats receiving a single daily dose of aminoglycoside have demonstrated less nephrotoxicity and less renal accumulation of the drug than rats receiving the same total daily dose by a multiple daily dosing schedule (3).

\section{COST CONTAINMENT}

The once-daily dosing of aminoglycosides would have a direct and immediate impact on both the cost of therapeutic drug monitoring and on the nursing and pharmacy time required for drug administration and preparation. Once-daily administration of aminoglycosides would eliminate the necessity for routine therapeutic drug monitoring of aminoglycoside levels.

\section{CLINICAL EFFICACY}

Twelve published studies have compared the oncedaily dosing of aminoglycosides with more conventional multiple-daily dosing regimens. Included in these studies is the recent publication of a randomized multicentre trial involving 677 patients receiving aminoglycosides for the treatment of febrile neutropenia (14). In all of these studies, once-daily dosing of aminoglycosides was as effective as and no more toxic than multiple-daily dosing of the drug.

A recent survey (15) at The Toronto Hospital indicated that of 71 serum aminoglycoside pairs only 18 (25.4\%) were appropriately performed with respect to collection time and its documentation. This observation underscores the need to scrutinize carefully the need for serum aminoglycoside levels. Taking into consideration conservative figures for the costs of serum aminoglycoside concentration determinations (personal communication), the numbers of levels done per year, and the costs of once-daily administration of gentamicin and tobramycin versus the actual costs of conventional (every 6, 8 or 12 h) dosing for one month and extrapolating these figures for one year, assuming no changes in risks or benefits, the cost savings for The Toronto Hospital are just over $\$ 100,000$. If the cost savings are expressed as a ratio and applied to six major Toronto teaching hospitals, the savings exceed \$0.5 million per annum.

Given the weight of the evidence available, should once-daily aminoglycoside therapy be adopted as a standard of practice? The answer to this question is, unequivocally, yes! The simplicity of dosing, maintenance (and possibly improvement) of clinical efficacy and potentially reduced toxicity, combined with minimal needs for serum aminoglycoside monitoring and the reduced costs associated with this practice, presents a powerful argument for its adoption into routine medical care. The Anti-Infectives Drug Products Advisory Committee of the Food and Drug Administration in the United States has recently concluded that there is sufficient information available to justify once-a-day dosing of aminoglycosides, and the agency is willing to consider changes in package inserts to reflect this. From our perspective we are presented with an opportunity for change with regard to aminoglycoside dosing, and it is a change that encompasses a territory well beyond the confines of the infectious diseases specialist. However, the implementation of this change in medical practice in Canada requires a committed leadership effort by the members of the infectious diseases community and others who are familiar with the concepts of aminoglycoside dosing. Let us seize the opportunity and move forward with this quality improvement effort.

\section{REFERENCES}

1. Davis BD. Mechanism of the bactericidal action of aminoglycosides. Microbiol Rev 1987;57:341-50.

2. Price KE. Aminoglycoside research 1975-1985: prospects for development of improved agents. Antimicrob Agents Chemother 1986;29:543-8.

3. Bennett WM, Wood CW, Houghton DC, Gilbert DN. Modification of experimental aminoglycoside nephrotoxicity. Am J Kidney Dis 1986;3:292-6.

4. Brummett RE, Fox KF. Aminoglycoside-induced hearing loss in human. Antimicrob Agents Chemother 1989:33:797-800.

5. Mathe H, Craig WA, Perchere PC. Determinants of efficacy and toxicity of aminoglycosides. J Antimicrob Chemother 1989:24:281-93.

6. Bennett WM, Plamp CE, Gilbert DN, Parker RA, Porter GA. The influence of dosage regimen on experimental gentamicin nephrotoxicity: dissociation of peak serum levels from renal failure. J Infect Dis 1979;140:576-80.

7. Labovitz E, Levison ME, Kaye D. Single dose daily gentamicin therapy in urinary tract infections. Antimicrob Agents Chemother 1974;6:465-70.

8. Powell SM, Thompson WL, Luthe MA, et al. Once-daily vs continuous aminoglycoside dosing: efficacy and toxicity in animal and clinical studies of gentamicin, and tobramycin. J Infect Dis 1983;147:918-32.

9. Gerber AU. Comparison of once-daily versus thrice-daily human equivalent dosing of aminoglycosides: basic considerations and experimental approach. J Drug Dev 1988; 1 (Suppl 3):17-23.

10. Vogelman B, Craig W. Kinetics of antimicrobial activity. J Pediatr 1986;5:835-40.

11. Carpenter T, Sande MA. Single large daily dosing versus intermittent dosing of tobramycin for treating experimental Pseudomonas pneumoniae. J Infect Dis 1988;158:7-12.

12. Vogelman BS, Craig WA. Post-antibiotic effects. J Antimicrob Chemother 1985;15:37-46.

13. Brummett RE, Fox KF. Aminoglycoside-induced hearing loss in humans. Antimicrob Agents Chemother 1989;33:797-800.

14. The International Antimicrobial Therapy Cooperative Group of the European Organization for Research and Treatment of Cancer. Efficacy and toxicity of single-daily doses of amikacin and ceftriaxone versus multiple daily doses of amikacin and ceftazidime for infection in patients with cancer and granulocytopenia. Ann Intern Med 1993; 1 19:584-93.

15. Seto R. An evaluation of the serum aminoglycoside level process at The Toronto Hospital. (Pharmacy Residency Project). Department of Pharmaceutical Services, The Toronto Hospital, 1993.

JM Conly MD FRCPC, W Gold MD FRCPC, SD Shafran MD FRCPC Departments of Medicine. The Toronto Hospital, University of Toronto, Toronto, Ontario and University of Alberta Hospital, University of Alberta, Edmonton. Alberta 


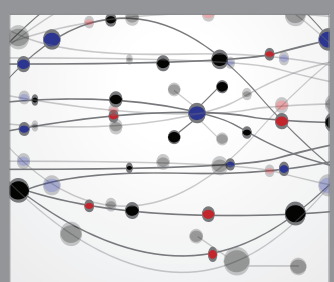

The Scientific World Journal
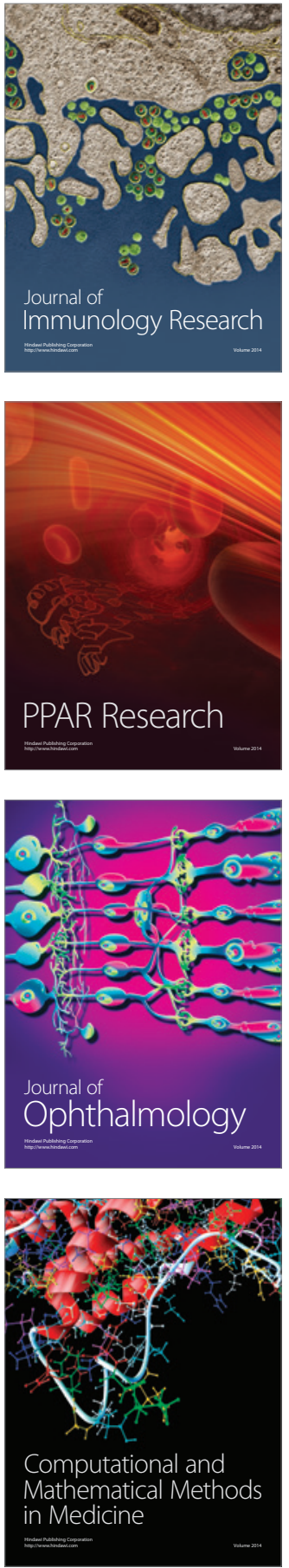

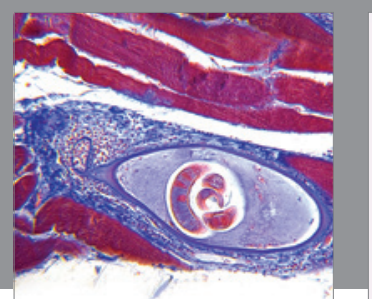

Gastroenterology Research and Practice

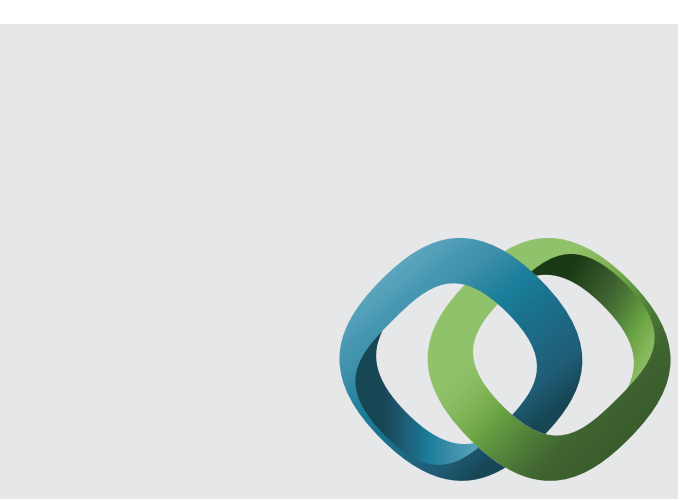

\section{Hindawi}

Submit your manuscripts at

http://www.hindawi.com
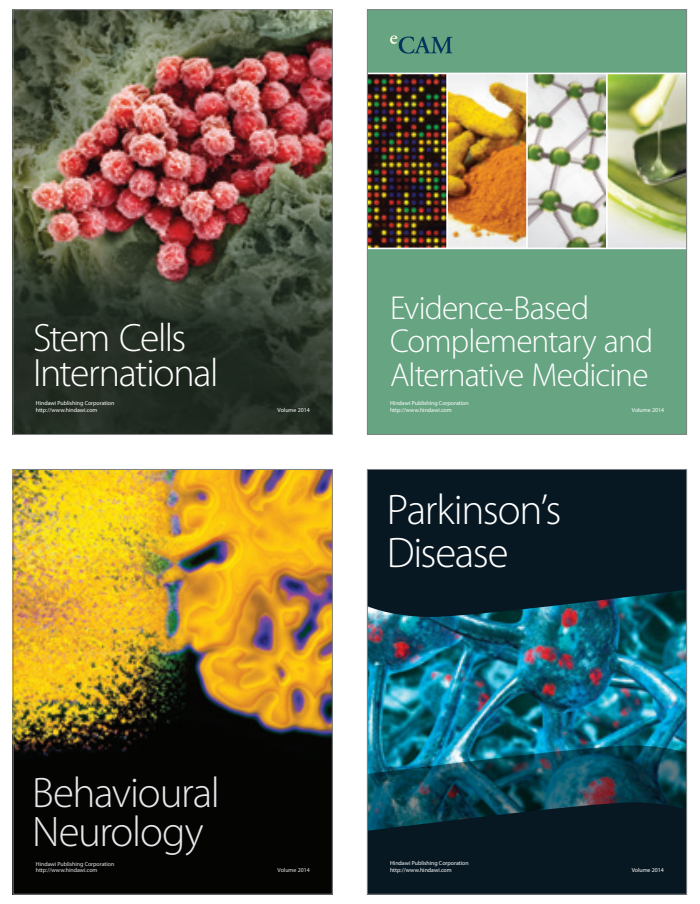
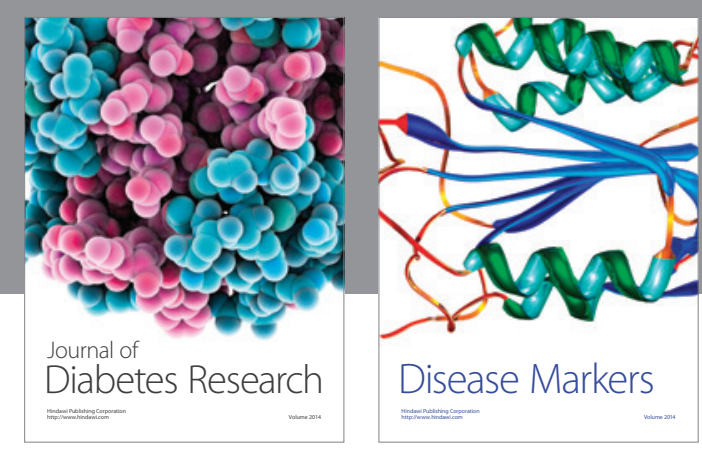

Disease Markers
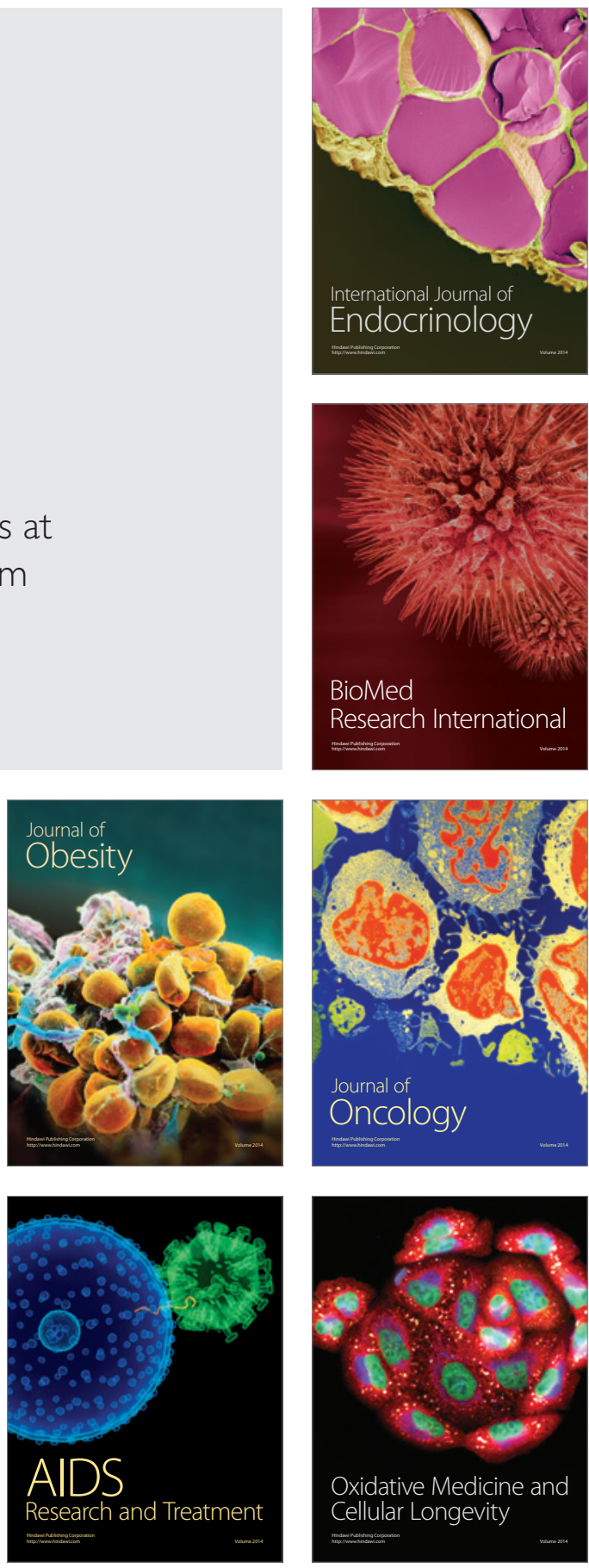